\title{
Has the role of exchange nailing in femoral nonunion diminished?
}

\author{
Vivek Trikha $\cdot$ Sanjay Yadav
}

Received: 3 August 2011 /Accepted: 17 August 2011 /Published online: 8 September 2011

(C) Springer-Verlag 2011

The article "Failed intramedullary nailing of femur: open reduction and plate augmentation with the nail in situ" by G.Z. Said, H.G. Said and M.M. El-Sharkawi, International Orthopaedics [1] evokes great interest and attention, as femoral nailing is a highly common procedure in orthopaedic training. The authors focussed particularly on open reduction of bone fragments and plate augmentation with nail in situ in rotational malalignment in nonunion cases. In their study, union was achieved in all nonunions treated with augmentation plate, which implies that augmentation plating can be one of the promising treatment options [2, 3]. However, the fact that $35.7 \%$ cases had unlocked nailing and $42.8 \%$ had unreamed nailing in the first instance cannot be ignored. Both these options are largely not recommended as the first treatment choice in femoral fractures, as they cannot provide the degree of stability that a static locked and reamed nail can provide. This re-emphasises the role of standard management protocols of a statically locked and reamed intramedullary nail as the primary treatment modality for femoral fracture [4]. This could have avoided various complications encountered, such as nonunion and malalignment in some if not all cases. In femoral nonunion after locked nail, the option proposed by the authors can be considered along with exchange nailing.

Another question that arises is whether the options of exchange nailing and occasional dynamisation have lost their relevance in the current scenario. Exchange nailing provides intramedullary reaming products that stimulate bone healing, whereas the use of a larger diameter nail can

V. Trikha $(\bowtie) \cdot$ S. Yadav

All India Institute of Medical Sciences,

Department of Orthopaedics,

New Delhi, India

e-mail: vivektrikha@rediffmail.com provide the additional stability required, especially in nonunion of the isthmus region. The role of augmentation plate may be better utilised in cases of distal femoral nonunions where adequate rotational stability may not be achieved by exchange nailing due to the soft cancellous bone in the wide metaphysis [3].

We also feel that the six to ten hole dynamic compression plate (DCP) used in the study are long plates requiring a longer incision than that of bone graft in isolation. As proposed, a locking compression plate (LCP) can be a better option than DCP, as smaller plates can be applied with the advantage of short, angular, stable screws, thus increasing rotational stability and cosmesis.

Finally, we reiterate that there is no substitute for a standard nailing - reamed and static locked - as primary surgery. Procedures such as exchange nailing are worth considering, especially in cases of femoral nonunions of the isthmus region. Augmentation plate with the nail in situ may offer another option in specific cases of distal femoral nonunions.

\section{References}

1. Said GZ, Said HG, El-Sharkawi MM (2011) Failed intramedullary nailing of femur: open reduction and plate augmentation with the nail in situ. Intern Orthop 35(7):1089-1092

2. Park K, Kim K, Choi YS (2011) Comparison of mechanical rigidity between plate augmentation leaving the nail in situ and interlocking nail using cadaveric fracture model of the femur. Int Orthop 35 (4):581-585

3. Park J, Kim SG, Yoon HK, Yang KH (2010) The treatment of nonisthmal femoral shaft nonunions with im nail exchange versus augmentation plating. J Orthop Trauma 24(2):89-94

4. Wild M, Gehrmann S, Jungbluth P, Hakimi M, Thelen S, Betsch M, Windolf J, Wenda K (2010) Treatment strategies for intramedullary nailing of femoral shaft fractures. Orthopedics 33(10):726. doi:10.3928/01477447-20100826-15 